\title{
Modeling of local disturbance formation in the ionosphere electron concentration before strong earthquakes
}

\author{
M. V. Klimenko ${ }^{1,2}$, V. V. Klimenko ${ }^{1}$, I. E. Zakharenkova ${ }^{1}$, and I. V. Karpov ${ }^{1}$ \\ ${ }^{1}$ N.V. Pushkov IZMIRAN RAS, West Department, Kaliningrad, 236010, Russia \\ ${ }^{2}$ Kaliningrad State Technical University, Kaliningrad, Russia
}

(Received May 31, 2010; Revised July 6, 2011; Accepted July 7, 2011; Online published July 27, 2012)

\begin{abstract}
In the past numerical experiments had shown that local disturbances of zonal electric fields allow reproducing the morphology of ionospheric disturbances during seismic active periods. In these numerical experiments the physical processes leading to the formation of such electric fields were not discussed. This paper offers two potential formation mechanisms of ionospheric precursors based on (1) the propagation and dissipation of smallscale internal gravity waves (IGWs) in the upper atmosphere, and (2) the penetration of a vertical electric field from the atmosphere into the ionosphere. We have simulated the effects of small-scale IGWs and the penetration of a vertical electric field for a mid-latitude earthquake in Greece using the Global Self-consistent Model of the Thermosphere, Ionosphere and Protonosphere (GSM TIP). It is shown that the simulation results obtained with the IGWs hypothesis are in good qualitative agreement with GPS TEC data. The simulation results obtained with the vertical electric field hypothesis are in less good agreement with GPS TEC data.

Key words: Ionosphere, electron density, small-scale Internal Gravity Waves, vertical electric field, zonal electric field, numerical modeling, GPS TEC data.
\end{abstract}

\section{Introduction}

The cumulative death toll due to strong earthquakes grows every year. Numerous scientists from different areas of geophysics are trying to construct an earthquake prediction theory that can be used to reduce the number of earthquake victims. Ionospheric researches have long been trying to identify earthquake precursors in the ionospheric electron density distribution. First investigations started after the 1964 Alaska earthquake (Davies and Baker, 1965; Leonard and Barnes, 1965), and they became more intensive in the last two decades (e.g., Pulinets and Boyarchuk, 2004; Liperovsky et al., 2008). Oraevsky et al. (2000), Liu et al. (2004), and Zakharenkova et al. (2006, 2007) have shown that before strong mid-latitude earthquakes the effects in total electron content (TEC) look like local changes in electron concentration with a maximum located in immediate proximity to the epicentre.

Numerical simulations with the Global Self-consistent Model of the Thermosphere, Ionosphere and Protonosphere (GSM TIP) (Namgaladze et al., 1988, 1991) show that observable ionospheric precursors of earthquakes can be reliably reproduced by the local disturbance of zonal electric fields (Namgaladze et al., 2009). It is necessary to note that the Namgaladze et al. (2009) paper does not discuss the physical processes leading to the formation of such zonal electric fields. Without a source mechanism, it is not reasonably possible to establish the cause-effect relations be-

Copyright (C) The Society of Geomagnetism and Earth, Planetary and Space Sciences (SGEPSS); The Seismological Society of Japan; The Volcanological Society of Japan; The Geodetic Society of Japan; The Japanese Society for Planetary Sciences; TERRAPUB

doi:10.5047/eps.2011.07.004 tween seismic events and ionospheric disturbances; however, one can estimate the quantitative characteristics of the electric fields necessary for the explanation of the observed disturbances.

In the current paper we present a careful analysis of the current conceptions of possible physical mechanisms responsible for the seismo-ionospheric effects formation. We show that small-scale Internal Gravity Waves (IGWs) and/or seismogenic electric fields in the Earth's ionosphere can be considered as the most probable formation mechanisms of TEC disturbances, observable prior to strong midlatitude earthquakes. This fact is also marked in Hayakawa (2007) and Klimenko et al. (2011). We present the GSM TIP model simulation results of the ionospheric response to the small-scale IGWs and/or seismogenic electric fields in the epicentral vicinity of the Greek earthquake of 8 January 2006. As observational data source the global GPS TEC maps were used in order to compare GSM TIP model simulation results with experimental data.

The primary task of this investigation consisted in testing of the efficiency of small-scale IGWs and seismogenic electric fields in the formation of the ionospheric disturbances prior to an earthquake. The formation mechanisms of IGWs and seismogenic electric fields near the earthquake epicenter and the possibility of their penetration from the lithosphere into the thermosphere and ionosphere were not investigated in the given paper.

\section{Lithosphere-Atmosphere-Ionosphere Cou- pling Prior to Earthquake}

The physical models of the lithosphere-atmosphereionosphere coupling have been extensively studied (e.g., 
Liperovsky et al., 1992; Pulinets and Boyarchuk, 2004; Hayakawa, 2007; Liperovsky et al., 2008). However, our knowledge of these coupling mechanisms is still limited. Pertsev and Shalimov (1996) proposed as a kind of mechanism the IGWs of seismogenic origin with the period $\sim 1-3$ hours generated due to non-stationary inflow of lithosphere gases into the atmosphere prior to an earthquake. This mechanism is able to explain the ionospheric phenomena, which occurred at large distances from the earthquake epicentre. Therefore such large-scale IGWs can not be considered as the formation mechanism of the ionospheric precursors located in immediate proximity from the earthquake epicentre. Mareev et al. (2002) and Molchanov (2004) considered the IGWs with periods from several minutes up to tens of minutes. The appearance of such waves makes it possible to attempt explaining the formation of seismoionospheric effects in the near epicentral area. The formation mechanism of earthquake ionospheric precursors by a seismogenic electric field with amplitudes from units up to tens of $\mathrm{mV} \mathrm{m}^{-1}$ (Chmyrev et al., 1989) is described in detail in Sorokin and Chmyrev (1999) and Pulinets and Boyarchuk (2004). The occurrence of seismogenic electric fields in the ionosphere is connected with vertical turbulent transfer of the charged aerosols injected in the atmosphere and radioactive substances (isotopes of radon) during the earthquake preparation time. Abnormal electro-magnetic fields and emissions have been considered as another possible mechanism (Hayakawa and Fujinawa, 1994; Hayakawa and Molchanov, 2002). Hayakawa (2007) noticed that this mechanism was found to be insufficient because of the weak intensity of lithosphere radio emissions (Molchanov et al., 1995).

The survey of the proposed mechanisms led to the conclusion that the small-scale IGWs and/or seismogenic electric fields in the Earth's ionosphere can be considered as formation mechanisms of the seismo-ionospheric effects that are observed prior to the strong mid-latitude earthquakes.

\subsection{The formation of the ionospheric precursors by} seismogenic electric fields

Namgaladze et al. (2007) assumed that the most probable formation mechanism of preseismic TEC anomalies (Zakharenkova et al., 2006, 2007) is the vertical transport of the $F_{2}$-region ionospheric plasma under the action of the zonal electric field. The geomagnetic conjugacy of the ionospheric precursors of earthquakes (Pulinets et al., 2003), effects in the equatorial anomaly the development of which is controlled by the zonal electric field (Depueva and Ruzhin, 1995; Zakharenkova et al., 2008), and the reduction of the electron temperature at an altitude of $600 \mathrm{~km}$ above the epicentre of a low-latitude earthquake (Oyama et al., 2008) are strong arguments in favour of this hypothesis. Besides, the analysis of results of model calculations of the ionospheric reaction on the action of the additional zonal electric field in the vicinity of the epicentre mid-latitudinal and nearequatorial earthquakes convincingly testifies in favour of this hypothesis (Namgaladze et al., 2007, 2009; Klimenko and Klimenko, 2008; Zolotov et al., 2008).

But how can such electric fields arise at ionospheric heights prior to earthquakes? This question remains open. Let's note that there are no experimental acknowledgements of zonal electric field penetration into the ionosphere from the atmosphere and lithosphere. At the same time, there is experimental evidence of vertical electric field occurrence at the Earth's surface (Rulenko, 2000) and in the Earth's ionosphere (Tzanis and Vallianatos, 2001) during the time of earthquake preparation. Grimalsky et al. (2003) analyzed the problems of the electric field penetration from the lithosphere into the ionosphere prior to earthquakes. Pulinets (2009a, b) discusses the recent understanding of the formation mechanism of a vertical electric field in the atmosphere and its penetration into the ionosphere.

In the present paper we consider the small-scale IGWs with small amplitude as the formation mechanism of zonal electric fields of seismogenic origin in the Earth's ionosphere. Yokoyama et al. (2004) used small-scale regional model and demonstrated that IGWs with speeds of $80 \mathrm{~m} \mathrm{~s}^{-1}$ can generate the polarizing electric fields in the ionosphere.

\subsection{The formation of ionospheric precursors by inter- nal gravity waves}

Many experimental data confirmed the strengthening of wave activity in the area over the earthquake epicentre (Miyaki et al., 2002; Rozhnoi et al., 2007). However reliable characterization of the excited waves has not been established in terms of periods and amplitudes. The purpose of our study is the testing of the efficiency of generation of large-scale disturbances in the upper atmosphere and ionosphere, and zonal electric fields, caused by strengthening of IGWs activity above an epicentre prior to an earthquake. Thus we assume that the IGWs generated in the area of the earthquake preparation should propagate practically vertically. Under this assumption its dissipation effects are directly located above the earthquake epicentre and correspond to the observations of ionospheric disturbances prior to strong earthquakes. The assumption of vertical IGWs propagation limits our analysis to only small-scale IGWs with frequencies near the 'Brunt-Vaisala' frequency. Upwards vertically propagating IGWs interact among themselves and with the background state of the atmosphere. Large-scale disturbances in the atmosphere are generated by these processes. The large-scale dynamic processes leading to the global change of thermospheric circulation and ionospheric conductivity change the dynamo electric field. The spatial scale of IGWs energy dissipation area will define the smaller scale of disturbances of thermospheric and ionospheric parameters. Earlier the efficiency of largescale disturbances in the thermosphere, owing to the dissipation of small-scale IGWs, was studied using as example the model of the disturbances generated by the solar terminator (Karpov and Bessarab, 2008). In the numerical experiment the disturbances of the atmosphere created by propagating IGWs can be taken into account in local variations of parameters on the lower boundary of the model over the epicentre area. It will allow simulating the changes of the state of the atmosphere and ionosphere that are created by local disturbances in atmospheric parameters on the lower boundary of the thermosphere with the period of $10 \mathrm{~min}$ and amplitude of the thermosphere wind of $10 \mathrm{~m} \mathrm{~s}^{-1}$. The disturbances of the temperature, density, and pressure can be calculated from polarization relations on the basis of the mentioned assumptions concerning the scales and ampli- 

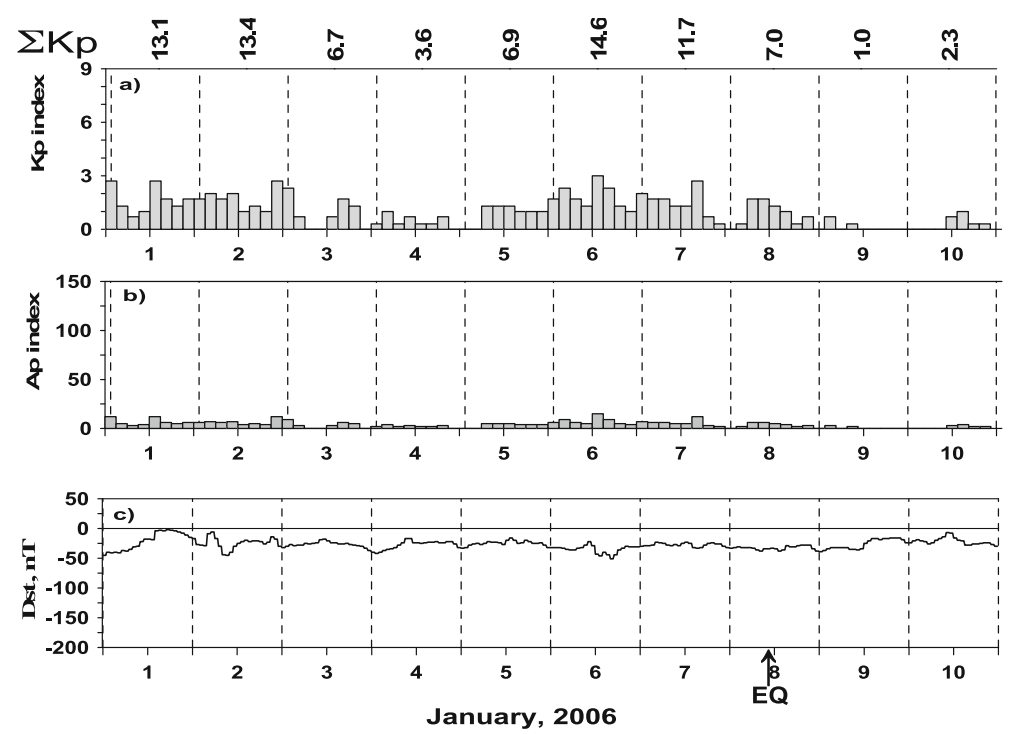

Fig. 1. Geomagnetic conditions for January 1-10, 2006. The behavior of $K_{\mathrm{p}}, A_{\mathrm{p}}$, and $D_{\mathrm{st}}$ indices of geomagnetic activity.

tudes of waves (Brjunelli and Namgaladze, 1988; Karpov and Bessarab, 2008). The selected typical period of IGWs (10 min) close to the period of 'Brunt-Vaisala' defines the vertical propagation of waves. The IGWs amplitude of $10 \mathrm{~m} \mathrm{~s}^{-1}$ does not exceed the perturbations of the wind observable in the lower thermosphere.

The IGWs period defines the altitude up to which these waves in the atmosphere can propagate. The increase in the period leads both to a reduction in altitude up to which the wave can propagate owing to its dissipation in the atmosphere, and to a displacement of the energy dissipation area owing to the occurrence of horizontally propagating IGWs. So the IGWs with the periods greater than $\sim 10$ min cannot vertically propagate. Results from model calculations with various wave periods and an explanation of the excitation mechanisms of large-scale disturbances owing to the smallscale IGWs dissipation are given by Karpov and Bessarab (2008).

We selected the IGW amplitudes at the lower boundary of the thermosphere based on the condition that they not exceed the wind velocity at these heights. Considering that the average wind speed and the amplitudes of tidal harmonics reach several tens of meters per second, we believe that the contribution to the general circulation from the selected IGWs at these heights will be insignificant. It is known that IGWs of tropospheric origin produce this kind of amplitudes. These amplitudes are also obtained by using a realistic simulation of the IGWs during the Sumatra tsunami (Occhipinti et al., 2006, 2008; Alam Kherani et al., 2009). Wind measurements in the lower thermosphere (MLT-radar for example) have measurement errors of $\sim 10 \mathrm{~m} \mathrm{~s}^{-1}$ (Forbes et al., 1999). A perturbation amplitude of wind velocity $10 \mathrm{~m} \mathrm{~s}^{-1}$ of the setting IGWs does therefore not exceed the measurement error. This suggests that small-scale IGWs (10 min) with such amplitude are impossible to identify in observations.

\section{Ionospheric Precursors of Earthquakes in GPS TEC}

In recent years preseismic and co-seismic ionospheric effects in GPS TEC measurements have been widely reported (e.g., Liu et al., 2001, 2004; Plotkin, 2003; Afraimovich et al., 2004; Zakharenkova et al., 2008). The GPS permanent network consists of more than 3000 GPS stations all over the world providing regular monitoring of the ionosphere on a global scale with reasonably high spatial and temporal resolution of TEC measurements. The global TEC maps are generated routinely by the IGS community with resolution of $5^{\circ}$ longitude and $2.5^{\circ}$ latitude and time interval of 2 hours.

In Oraevsky et al. (2000), Liu et al. (2004), and Zakharenkova et al. (2006, 2007), the authors demonstrate that for strong middle-latitude earthquakes, the preseismic effects in TEC look like the local increase/decrease of electron density which can be observed 2-3 days prior to a strong earthquake, and the maximum zone of these disturbances is located in the immediate vicinity of the epicenter. The spatial dimension of the seismo-ionospheric anomaly can reach several 1000 kilometres in longitude and about $1000-1500 \mathrm{~km}$ in latitude. When approaching the beginning of the earthquake the amplitudes of the anomaly increase up to $40-100 \%$ relative to the background level.

The detailed analysis of GPS TEC variations prior to the strong Greece earthquake ( $M$ 6.8) that took place on 8 January 2006 can be found in Zakharenkova et al. (2008). In Fig. 1 the variations of geomagnetic activity indices $\left(K_{\mathrm{p}}\right.$, $A_{\mathrm{p}}$, and $D_{\text {st }}$ ) for the period of 1-10 January 2006 are presented. It is evident that all indices indicate quiet geomagnetic conditions; $\Sigma K_{\mathrm{p}}$ values did not exceed a level of 20 .

Figure 2 shows the vertical TEC measurements for the five previous days and one day after the earthquake, and the median TEC data calculated for the considered period above separate GPS stations located in the immediate vicinity of the earthquake region. The analysis of daily variations shows that one day prior to the main event (on 7 January) a significant increase of TEC values was observed at all sta- 
$\operatorname{ORID}\left(41.07^{\circ} \mathrm{N}, 20.47^{\circ} \mathrm{E}\right)$

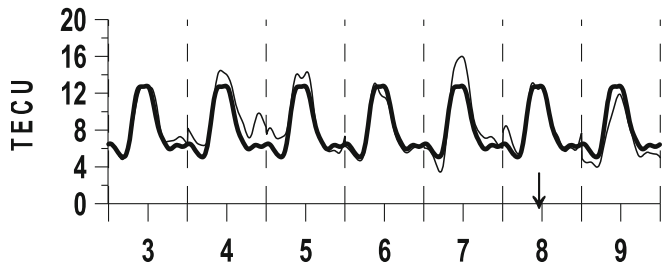

TUBI $\left(40.47^{\circ} \mathrm{N}, 29.27^{\circ} \mathrm{E}\right)$

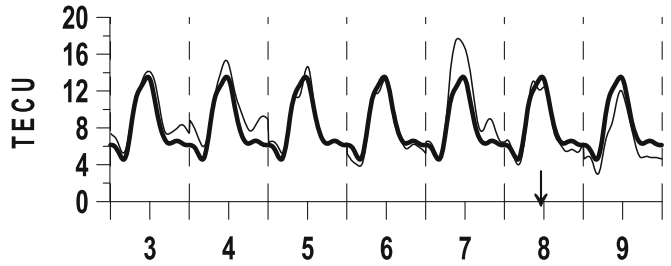

$\operatorname{MATE}\left(40.39^{\circ} \mathrm{N}, 16.42^{\circ} \mathrm{E}\right)$

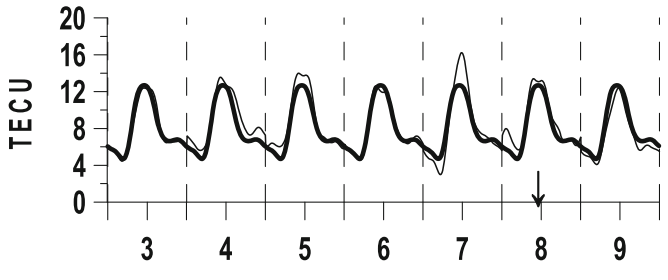

NOT1 $\left(36.52^{\circ} \mathrm{N}, 14.59^{\circ} \mathrm{E}\right)$

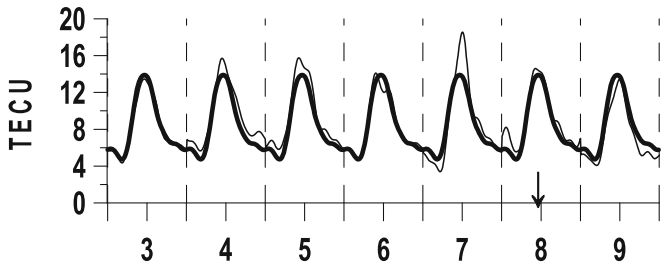

Fig. 2. Daily TEC variations above stations ORID, TUBI, MATE, and NOT1 for the period 3-9 January $2006\left(1 \mathrm{TECU}=10^{16} \mathrm{el} \mathrm{m}^{-2}\right)$. Thin lines are current variations of TEC, and thick lines are median values. The arrows note the moment of the earthquake.

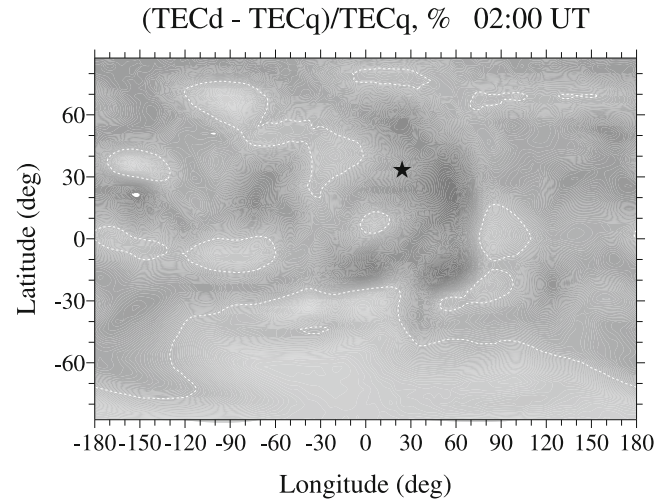

(TECd - TECq)/TECq, \% 14:00 UT

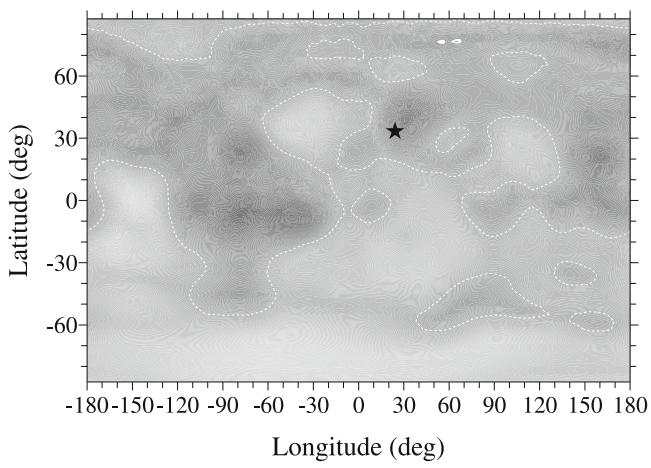

$(\mathrm{TECd}-\mathrm{TECq}) / \mathrm{TECq}, \% \quad 10: 00 \mathrm{UT}$

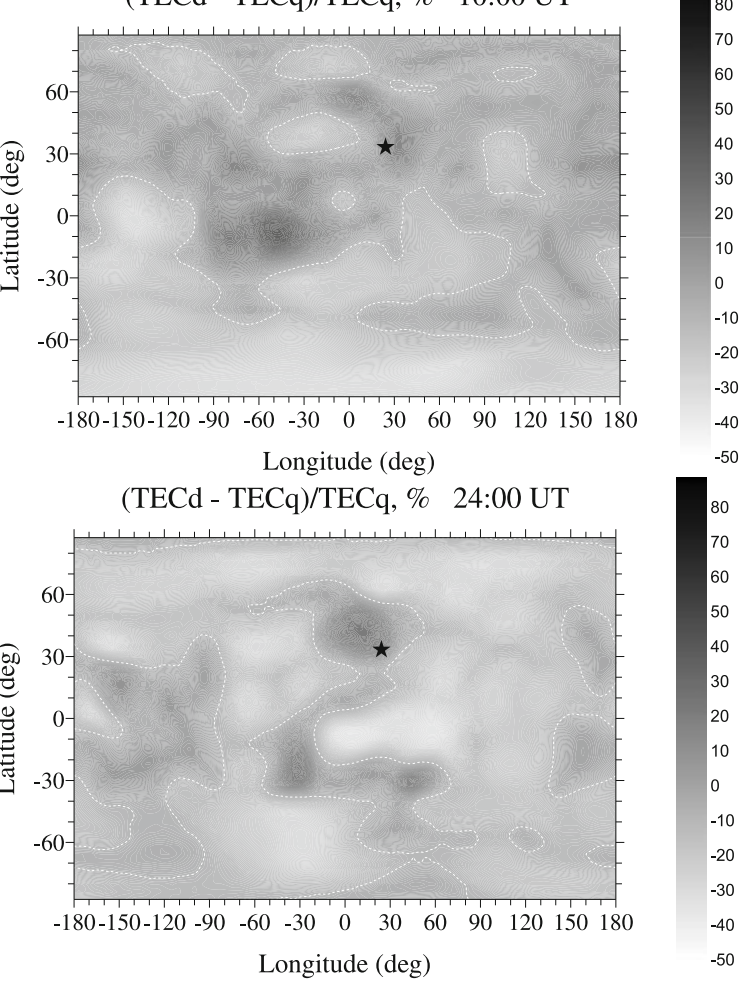

Fig. 3. Global maps of TEC deviations prior to the strong earthquake in Greece plotted on the basis of GPS TEC data. The asterisk shows the position of the earthquake epicenter. White dotted lines show the boundaries between positive and negative disturbances.

tions. This increase reached the value of 5.5 TECU which is more than twice the level of the "day-to-day" variability for quiet periods. Other days of the considered period were characterized by rather weak deviations of the current TEC values from the median.

In order to estimate the spatial scales and temporal dynamics of the seismo-ionospheric anomaly the global GPS TEC maps had been analyzed. Figure 3 demonstrates the differential TEC maps for the times 02:00, 10:00, 14:00, and 24:00 UT. It is evident that at 02:00 and 24:00 UT positive disturbances in TEC are observed both in epicentral and in magneto-conjugated areas, whereas at 10:00 and
14:00 UT the positive disturbances in TEC are observed only in the epicentral area. Note that in the first case the epicenter of the earthquake is on the night side, while it is on the dayside in the second case. It needs to be mentioned that positive and negative variations of TEC occur all over the globe and not only over the epicentral area.

\section{Numerical Modelling of the Earthquake Pre- cursors by the GSM TIP Model}

The present paper describes the modelling of the ionospheric response on the action of the IGWs and seismogenic electric fields set in the vicinity of the middle-latitude 

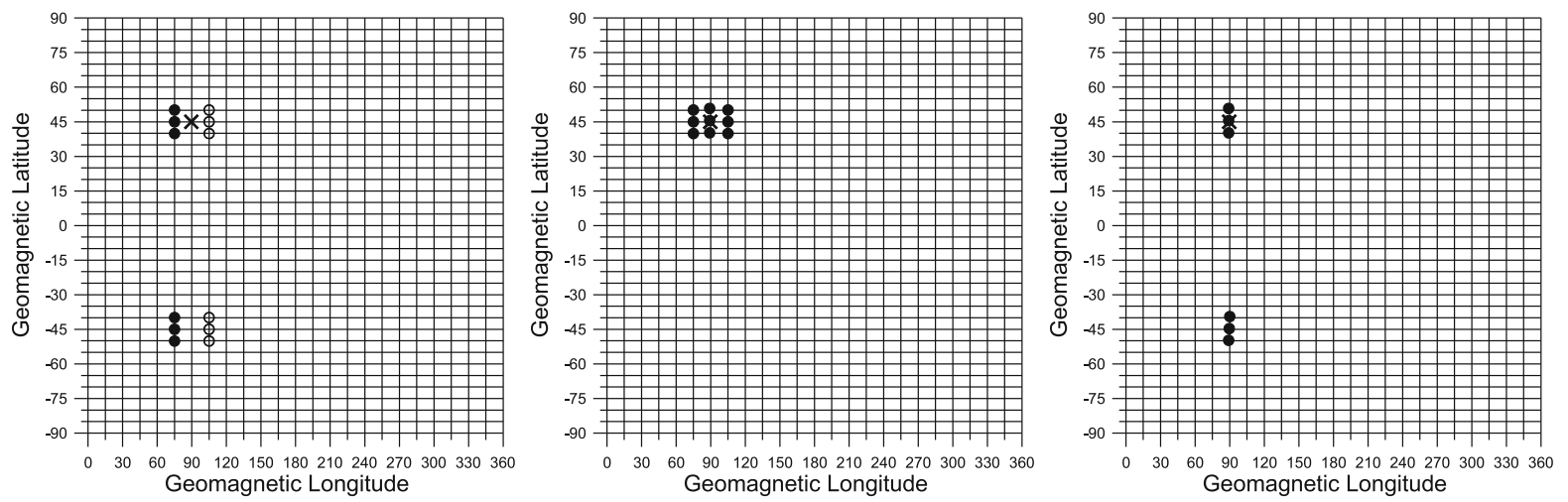

Fig. 4. The spatial grids of the model GSM TIP in geomagnetic coordinates for setting of the different sources of ionospheric disturbances before the strong earthquake in Greece. The methods of setting of these sources: at the left—eastward electric field at height of $175 \mathrm{~km}$ (dark and light points - grid points with additional positive and negative potential $10 \mathrm{kV}$ ); in the middle — the IGWs at height of $80 \mathrm{~km}$ (circles mark grid points with IGWs); on the right—vertical electric field penetrated into the ionosphere at height of $175 \mathrm{~km}$ (circles mark grid points with $10 \mathrm{kV}$ potential).

earthquake epicentre in Greece. The calculations were carried out using the GSM TIP model (Namgaladze et al., 1988, 1991) developed in WD IZMIRAN (West Department of Pushkov Institute of Terrestrial Magnetism, Ionosphere and Radio wave propagation RAS). The calculation of electric fields of dynamo and magnetospheric origin has recently been modified by Klimenko et al. (2006, 2007). The GSM TIP model calculates the time-dependent global three-dimensional distributions of the neutral temperature, composition $\left(\mathrm{O}_{2}, \mathrm{~N}_{2}, \mathrm{O}\right)$, and velocity vector of neutral gas, as well as the density, temperature, and velocity vectors of atomic $\left(\mathrm{O}^{+}, \mathrm{H}^{+}\right)$and molecular $\left(\mathrm{N}_{2}^{+}, \mathrm{O}_{2}^{+}, \mathrm{NO}^{+}\right)$ions and electrons. Additionally, the model also provides the $2 \mathrm{D}$ distribution of the potential of the electric field of the dynamo and magnetosphere origin.

The model input parameters are the following: concentration of neutral hydrogen; the maximal stream of energy of photoelectrons; EUV and UV fluxes of the solar radiation; precipitations of high-energy particles; amplitudes and spatial distribution of Region 1 field aligned currents or a cross-polar cap potential difference and Region 2 field aligned currents; year; month; day; the level of solar and geomagnetic activity. The Earth's magnetic field is approximated by the tilted dipole, taking into account the difference of geographical and geomagnetic axes of the Earth.

In Fig. 4 the global spatial grids of the model GSM TIP in geomagnetic coordinate system are shown for two different methods that let us set the seismogenic source of ionospheric disturbances before the strong earthquake event in Greece. This seismogenic source was set and did not change within 24 hours. The first method consists of a set of eastward electric fields in the epicentral vicinity and is described in Namgaladze et al. (2009). In this method the additional positive and negative electric potentials equal to the absolute value of $10 \mathrm{kV}$ were set in the grid points on the east and west boundaries of the epicentral area at the height of $175 \mathrm{~km}$. We consider that the epicentral region extends $10^{\circ}$ in latitude and $30^{\circ}$ in longitude, which approximately corresponds to the horizontal extensions of the regions with anomalous pre-earthquake TEC enhancements (e.g., Zakharenkova et al., 2007, 2008). The spatial scale of the additional longitudinal eastward electric field is $3000 \mathrm{~km}$, and the meridional extent is about $1000 \mathrm{~km}$. Namgaladze et al. (2009) found that the values of the additional eastward electric fields prior to earthquakes are larger than those of the background undisturbed electric fields (an increase in the zonal electric field over the earthquake epicenter of $\sim 4-10 \mathrm{mV} \mathrm{m}^{-1}$ ). However, these values are substantially smaller than the characteristic values of the highlatitude electric field of magnetospheric origin under quiet conditions $\left(\sim 15-25 \mathrm{mV} \mathrm{m}^{-1}\right)$. Owing to the closed geomagnetic field lines at heights of the ionospheric $F$-region and higher, the set of additional potentials near the epicentral area leads to the occurrence of the same potentials in the magneto-conjugate area.

The second method consists of a set of IGWs in the chosen grid points over the epicentral vicinity. In our calculations we did not use any special model of IGWs propagation. In the GSM TIP model the disturbed environment related to IGWs are set only on the lower boundary of the thermosphere at a height of $80 \mathrm{~km}$ in the vicinity of the earthquake epicenter. Thermospheric and ionospheric dynamics are calculated from the three-dimensional nonlinear equations of hydrodynamics, i.e., equations of continuity, movement, and energy, which describe the conservation laws of thermal plasma in the upper atmosphere of the Earth. It is assumed that the IGWs propagate upward from an altitude of $80 \mathrm{~km}$ and generate the large-scale disturbances in the upper atmosphere. In the calculations the IGWs with the period of $10 \mathrm{~min}$ and amplitude of $10 \mathrm{~m} \mathrm{~s}^{-1}$ were set at the bottom boundary of the thermosphere using the following formulas:

$$
\begin{aligned}
& \Delta n\left(\mathrm{O}_{2}\right)=n\left(\mathrm{O}_{2}\right)_{0} \times A \times 10^{-2} \times \sin (2 \times \pi \times t / \tau), \mathrm{m}^{-3} \\
& \Delta n\left(\mathrm{~N}_{2}\right)=n\left(\mathrm{~N}_{2}\right)_{0} \times A \times 10^{-2} \times \sin (2 \times \pi \times t / \tau), \mathrm{m}^{-3} \\
& \Delta n(\mathrm{O})=n(\mathrm{O})_{0} \times A \times 10^{-2} \times \sin (2 \times \pi \times t / \tau), \mathrm{m}^{-3} \\
& \Delta T_{n}=T_{n 0} \times A \times 4 \times 10^{-3} \times \sin (2 \times \pi \times t / \tau), \mathrm{K} \\
& \Delta V_{n \Theta}=A \times v_{0} \times \sin (2 \times \pi \times t / \tau), \mathrm{ms} \mathrm{s}^{-1} \\
& \Delta V_{n \Lambda}=A \times v_{0} \times \sin (2 \times \pi \times t / \tau), \mathrm{m} \mathrm{s}^{-1}
\end{aligned}
$$

where $A=10, v_{0}=1 \mathrm{~m} \mathrm{~s}^{-1}, A \times v_{0}$-amplitude of internal gravity wave, $\tau=600 \mathrm{~s}$-period of internal gravity wave, $t$-Universal Time in $\mathrm{s}, n\left(\mathrm{O}_{2}\right)_{0}=7.4 \times 10^{19} \mathrm{~m}^{-3}$, 
$n\left(\mathrm{~N}_{2}\right)_{0}=3.0 \times 10^{20} \mathrm{~m}^{-3}, n(\mathrm{O})_{0}=2.4 \times 10^{16} \mathrm{~m}^{-3}, T_{n 0}=$ $188 \mathrm{~K}$-background values of concentration of molecular oxygen, molecular nitrogen, atomic oxygen and temperatures of neutral gas, accordingly, on the bottom boundary of the thermosphere. The amplitude perturbations in the neutral density and temperature were calculated according to the relations of the IGWs linear theory found in Hines (1960).

The third method consists of a set of electric potential irregularity of $10 \mathrm{kV}$ in grid points of the epicentral area at a height of $175 \mathrm{~km}$. By setting such electric potential irregularity we model the penetration of a vertical electric field from the atmosphere into the ionosphere. Just as in the first method, this method also leads to the occurrence of the same potential in magneto-conjugated points. Besides, the model calculations with the setting of superposition of IGWs and vertical electric field were carried out.

These methods allow the generation of large-scale disturbances in the upper atmosphere and ionosphere caused by seismogenic sources over an epicentre prior to the earthquake. The results of the model calculation analysis are demonstrated through the global maps of TEC deviations. These TEC deviations were obtained by comparison of calculation results with and without taking into account the seismogenic sources as the model input parameters.

\section{Calculation Results and Discussion}

To study the effects in TEC we have plotted the calculated daily variations in TEC, zonal and meridional electric field, meridional thermospheric wind, and ratio $n(\mathrm{O}) / n\left(\mathrm{~N}_{2}\right)$ at the altitude of $300 \mathrm{~km}$ above the epicenter of the earthquake in Greece (Fig. 5). These results have been obtained using the GSM TIP model in the absence of seismogenic sources, and also by including in the epicentral area the following seismogenic sources: eastward electric field, IGWs, vertical electric field, and the superposition of IGWs and the vertical electric field.

It is seen that an eastward electric field causes a large positive disturbances in TEC above the earthquake's epicenter. On the other hand, the small reduction in $n(\mathrm{O}) / n\left(\mathrm{~N}_{2}\right)$ has no essential influence on the TEC behavior. At the same time, the additional meridional plasma drift under the influence of an eastward electric field causes an additional thermospheric wind directed poleward due to ion-neutral collisions. This wind slightly weakens the effects of the eastward electric field in TEC. The meridional electric field causes a zonal plasma drift at those heights where the plasma is magnetized possibly leading to longitudinal gradients of the electron density. At mid-latitudes they occur, mainly, in the vicinity of the morning and evening terminators. The obtained behavior of the additional meridional component of the electric field shows that this component leads to the weakening of the positive effects in TEC in the vicinity of the evening terminator, to the strengthening in the vicinity of the morning terminator, and to the insignificant amplification in the afternoon.

The setting of IGWs and vertical electric field leads to practically the same positive disturbances in TEC above the earthquake's epicenter. The TEC effect of the superposition of these mechanisms is equal to the effect of each mecha- nism taken separately. TEC disturbances above the epicenter, obtained at the setting of various seismogenic sources including an eastward electric field, are in satisfactory qualitative agreement with experimental data of GPS TEC observations one day prior to the earthquake in Greece, as shown in Fig. 2. The absolute values of TEC obtained in the model calculations are smaller than those observed above different stations located in the vicinity of the earthquake's epicenter. The reason for this is the overestimated neutral atmosphere density in the GSM TIP model that leads to overestimated rates of ionization losses in the ionospheric $F$-region. This in turn leads to the underestimated values of ionospheric electron density. However, the goal of this paper is the research of the ionospheric disturbances related to the earthquake preparation, instead of the absolute values of ionospheric parameters. It should be noted that the relative disturbances in TEC obtained in calculated results and from observational data are very similar.

The setting of IGWs leads to small changes in both components of the electric field. Small-scale fluctuations with the period of $10 \mathrm{~min}$, as well as IGWs, are very well seen in the meridional electric field. A source of the electric field changes is the IGW effects in the thermosphere at heights of the lower ionosphere, i.e., in the height range of 80-175 km. The IGW's dissipation at these heights leads to the change of thermospheric circulation and ionospheric conductivity. These changes in a current-carrying layer of the ionosphere lead to the change of a dynamo electric field. It should be noted that by using the obtained changes in electric field it is impossible to explain the positive disturbance in TEC obtained in the calculations. However, the additional eastward electric field makes the contribution to positive disturbance in TEC primarily in the afternoon, but this contribution is insignificant. In the interval 01:00-07:00 UT the additional equatorward wind should cause a positive disturbances in TEC, but there is also a small additional poleward wind that should lead to a insignificant negative effects in TEC. Figure 2 shows that $n(\mathrm{O}) / n\left(\mathrm{~N}_{2}\right)$ grows significantly. The neutral atmosphere composition changes affect the behavior of the electron density in the ionospheric $F$-region as follows. As the atomic oxygen is the basic source of the ionization at heights of the ionospheric $F$-region, and the molecular nitrogen is the basic source of the recombination, the growth of the ratio $n(\mathrm{O}) / n\left(\mathrm{~N}_{2}\right)$ leads to a positive disturbances in the electron density at $F$-region heights and in TEC. Thus, at the setting of IGWs the positive disturbances in TEC are caused, mainly, by the growth of the ratio $n(\mathrm{O}) / n\left(\mathrm{~N}_{2}\right)$.

Setting of a vertical electric field penetration from the atmosphere into the ionosphere as the seismogenic source leads to the occurrence of an eastward electric field. This electric field causes the formation of a positive disturbance in TEC. The variations of the meridional electric field in the post-sunset period counteract the formation of a positive disturbance in TEC. Changes of neutral atmosphere composition are practically absent, and the arising additional poleward wind prevents formation of positive disturbances in the afternoon.

Lastly, the setting of the superposition of IGWs and the vertical electric field affects the variations of neutral atmo- 

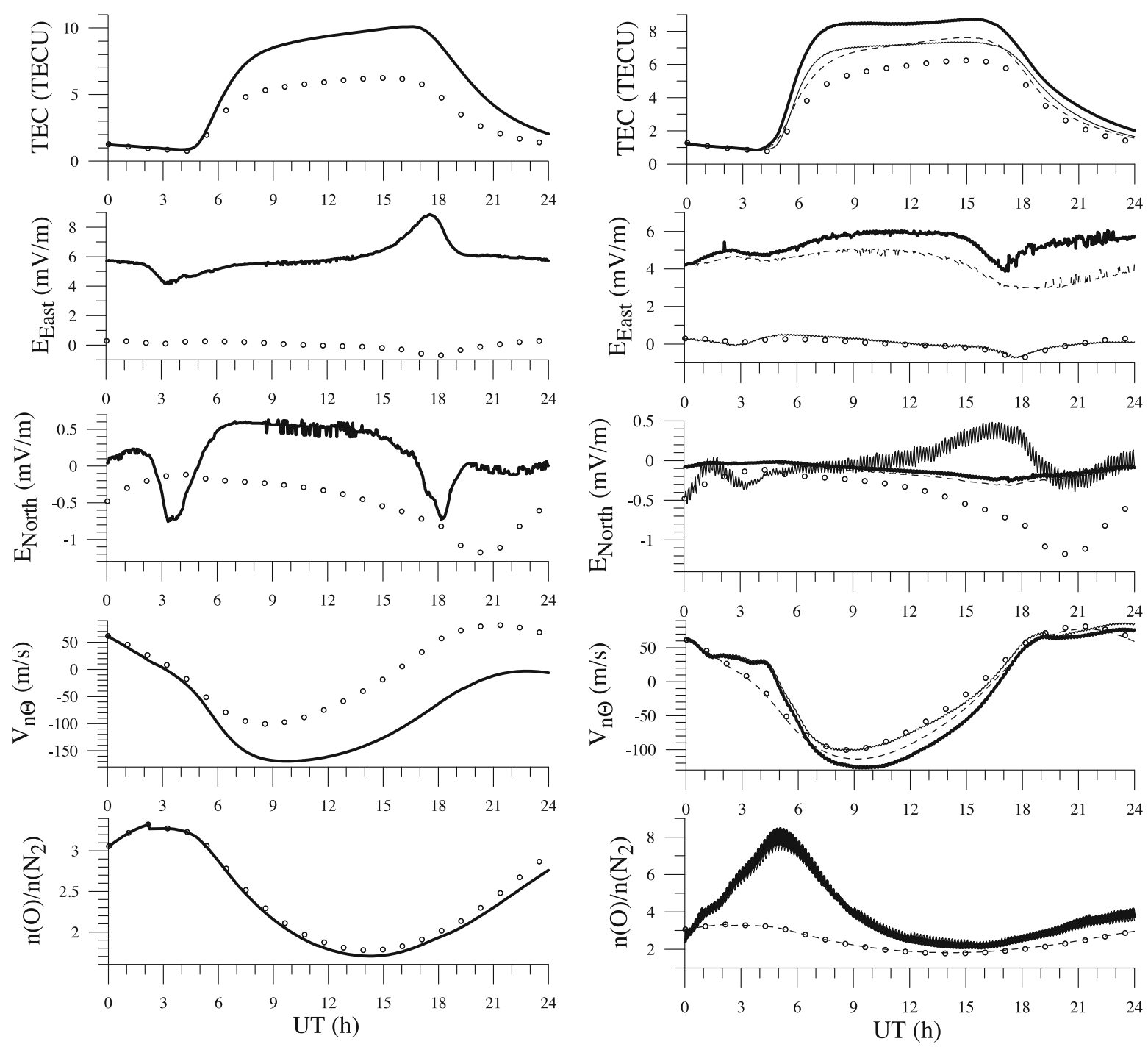

Fig. 5. The behavior of the TEC, zonal and meridional components of the electric field, meridional component of the thermospheric wind, and the ratio $n(\mathrm{O}) / n\left(\mathrm{~N}_{2}\right)$ in the vicinity of the Greece earthquake epicenter obtained in the model GSM TIP. The left panel—calculation results at the setting of additional eastward electric field (solid lines). The right panel—calculation results at the setting of potential irregularity connected with vertical electric field (dotted lines), IGWs (solid thin lines) and the superposition of these two mechanisms (solid thick lines). Light circles—quiet conditions.

sphere composition just as the IGWs itself, and the variations of the meridional electric field just as the vertical electric field itself. Note that the effects in meridional thermospheric wind and in zonal electric field are larger than the total effect of each of these mechanisms taken separately. The positive disturbance in TEC in this case is formed by the zonal electric field and by the growth of the ratio $n(\mathrm{O}) / n\left(\mathrm{~N}_{2}\right)$, and-in pre-sunrise hours-is supported by an additional equatorward wind. An additional poleward wind in the afternoon prevents the growth of TEC, and-in the vicinity of the evening terminator-the meridional electric field prevents any significant growth of TEC.

The sources of the meridional thermospheric wind and the corresponding variation for three different mechanisms are different. IGWs lead to the appearance of disturbances in the thermospheric wind due to changes in the neutral atmosphere composition. In the zonal/vertical electric field mechanisms, the meridional component of the thermospheric wind variation is caused by ion-neutral drag at the time of plasma $E \times B$-drift in the meridional direction. The plasma motions in the meridional direction are caused by the zonal electric field. The zonal electric field variations are the biggest at the setting of seismogenic zonal electric field mechanism.

Within the model calculations (Section 4) by using the GSM TIP model we have obtained global maps of TEC deviations with 1 hour time resolution. These global maps of TEC deviations for the different UT moments, obtained by taking into account the IGWs, the vertical electric field, and their superposition over the epicentral area are shown in Fig. 6. It can be seen that the calculations of TEC deviations including the IGWs are in better agreement with the experimental data one day prior to the earthquake in Greece, which are shown in Fig. 3: identity of the presence and absence of effects in the geomagnetic conjugate area to the earthquake epicentre in calculations and in experiment; good qualitative similarity of the global picture of the disturbances between model calculations and observations; quantitative agreement of local TEC disturbances in the epicentre area; the presence of significant dis- 

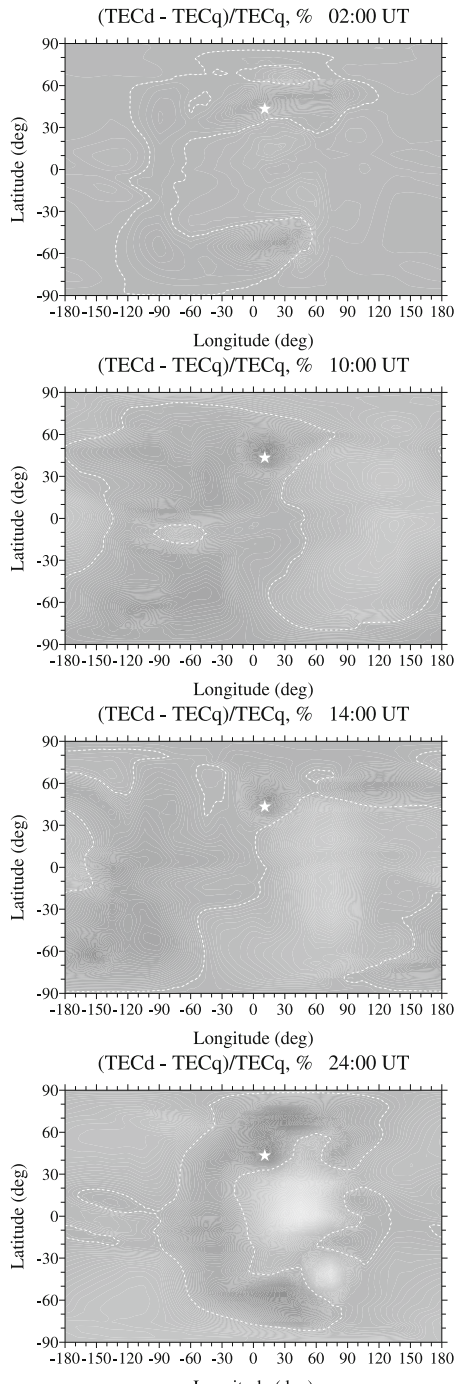
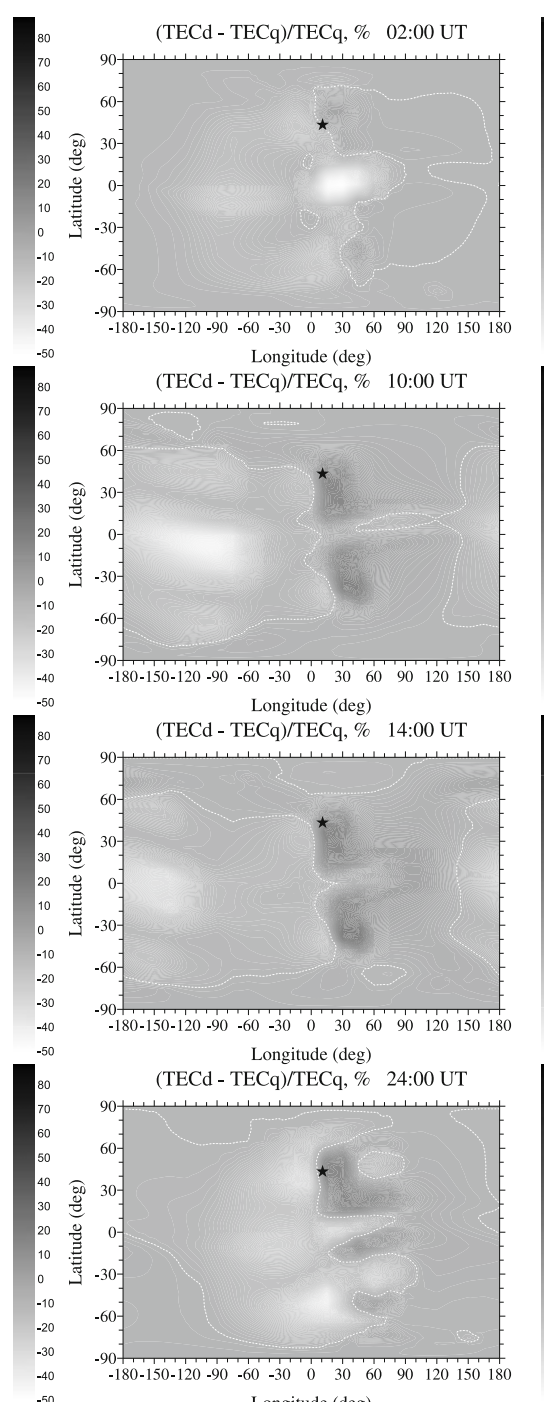
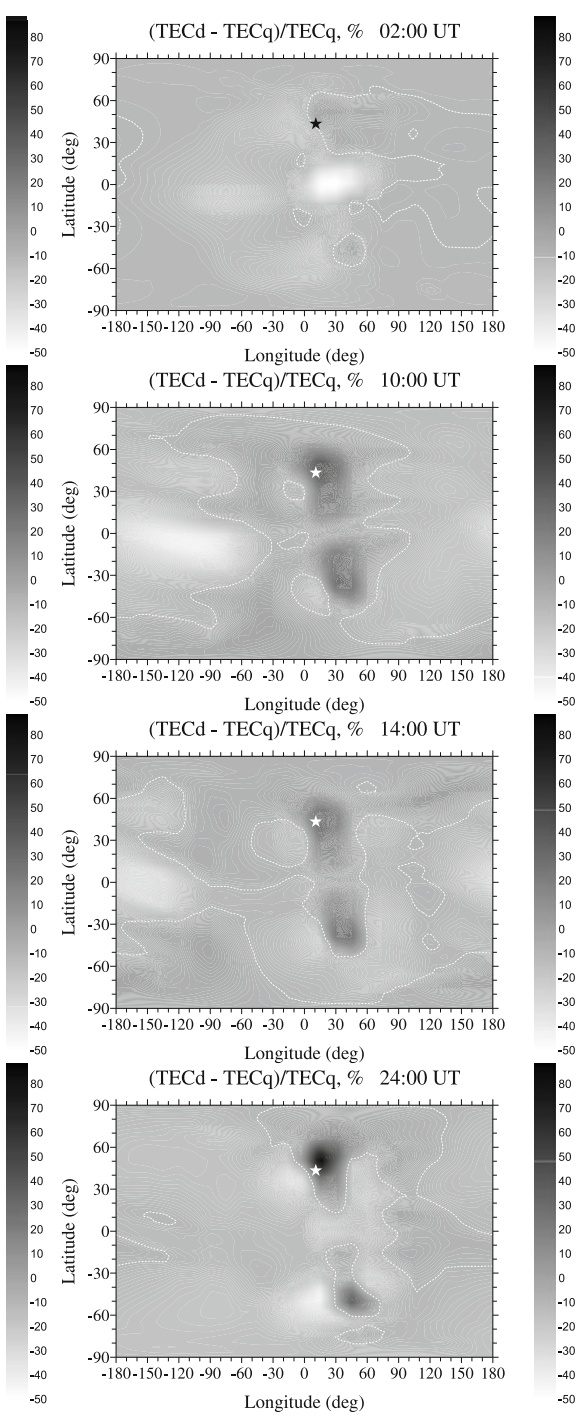

Fig. 6. Calculated model GSM TIP global maps of TEC deviations caused by the action of small-scale IGWs (left panel), vertical electric field (middle panel), and superposition of IGWs and vertical electric field (right panel) setting in the Greece earthquake epicentral area. The asterisk shows the position of an earthquake epicenter. White dashed lines show the boundaries between positive and negative disturbances.

turbances at the geomagnetic equator prior to the strong middle-latitude earthquake both in the model and the observations. This agreement confirms a correct choice of the small-scale IGWs as the formation mechanism of the ionospheric disturbances before the strong mid-latitude earthquake in Greece.

The effects in TEC of the vertical electric field and its superposition with IGWs are stronger than the effects obtained by only including IGWs. However, the use of the electric field as the formation mechanism for seismo-ionospheric effects leads to the obligatory occurrence of TEC positive disturbances in the area of magnetoconjugated to the earthquake epicentral area that was not consistently observed before the Greek earthquake.

\section{Summary and Conclusion}

Recent results of numerical simulations have demonstrated, that the local disturbances in zonal electric fields allow reproducing the morphology of the pre-seismic ionospheric effects. It is important to note that in previous numerical experiments (Namgaladze et al., 2007, 2009;
Zolotov et al., 2008) the physical processes leading to the formation of such electric fields were not discussed. In the given paper we proposed the formation mechanisms of the seismogenic zonal electric fields in the Earth's ionosphere by the propagation and dissipation of small-scale IGWs in the upper atmosphere and/or by the penetration of vertical electric field from the atmosphere into the ionosphere. We used numerical simulations with the goal of reproducing the changes in GPS TEC that were observed one day prior to the strong mid-latitude earthquake in Greece on 8 January 2006. For this purpose we considered several variants of the seismogenic source inputs at the epicentral area: a) zonal electric field; b) small-scale IGWs; c) penetration of vertical electric field; d) superposition of b) and c).

Our simulations revealed that TEC disturbances above the epicenter, caused by various seismogenic sources are in satisfactory qualitative agreement with experimental data of GPS TEC measurements. It was shown that small-scale IGWs were able to produce disturbances of the zonal electric field over the epicentral area. However the amplitude of these disturbances does not reproduce the observed 
local TEC deviations. Simulation results for small-scale IGWs demonstrate that the local positive effect in TEC over the epicentral area is related to the growth of the ratio $n(\mathrm{O}) / n\left(\mathrm{~N}_{2}\right)$. When the penetration of the vertical electric field from the atmosphere into the ionosphere is selected as the seismogenic source, the positive disturbance in TEC is formed as a result of the action of the additional eastward electric field.

The comparative analysis of the global TEC distributions produced by different seismogenic sources produced important results. It was revealed that the simulated maps of TEC deviations obtained by the setting of IGWs have the best agreement with the experimental data for different UT epochs. This result confirms that the presence of smallscale IGWs was the formation mechanism of the seismoionospheric effects for the earthquake under consideration.

It is possible to achieve better agreement between simulation results and observations by changing the amplitude and by combining different seismogenic sources. Our simulation analysis of ionospheric earthquake precursors for the January 2006 earthquake in Greece demonstrates the importance of comparing the results with experimental data. Investigations of the ionospheric behaviour over different seismo-active regions during quiet and disturbed conditions will require further research.

Acknowledgments. The authors are grateful to the IGS community for providing GPS permanent data and to the USGS Earthquake Hazards Program for the detailed earthquake information. We express our gratitude to B. Reinisch, E. Astafieva, P. Coïsson, F. Carlos de Meneses Jr., and A. Hasbi for their help in evaluating this paper.

\section{References}

Afraimovich, E. L., E. I. Astafieva, M. B. Gokhberg, V. M. Lapshin, V. E. Permyakova, G. M. Steblov, and S. L. Shalimov, Variations of the total electron content in the ionosphere from GPS data recorded during the Hector Mine earthquake of October 16, 1999, California, Russ. J. Earth Sci., 6, 339-354, 2004.

Alam Kherani, E., P. Lognonné, N. Kamath, F. Crespon, and R. Garcia, Response of the ionosphere to the seismic trigered acoustic waves: Electron density and electromagnetic fluctuations, Geophys. J. Int., 176, 1-13, 2009.

Brjunelli, B. E. and A. A. Namgaladze, Fizika Ionosfery, Moscow, Nauka, 1988 (in Russian).

Chmyrev, V. M., N. V. Isaev, S. V. Bilichenko, and G. A. Stanev, Observation by space-borne detectors of electric fields and hydromagnetic waves in the ionosphere over one earthquake center, Phys. Earth Planet. Inter., 57, 110-114, 1989.

Davies, K. and D. M. Baker, Ionospheric effects observed around the time of the Alaskan earthquake of March 28, 1964, J. Geophys. Res., 70, 2251-2253, 1965.

Depueva, A. Kh. and Yu. Ya. Ruzhin, Seismoionospheric fountain-effect as analogue of active space experiment, Adv. Space Res., 15, 1215112154, 1995.

Forbes, J. M., Yu. I. Portnyagin, N. A. Makarov, S. E. Palo, E .G. Merzlyakov, and X. Zhang, Dynamics of the lower thermosphere over South Pole from meteor radar wind measurements, Earth Planets Space, 51, 611-620, 1999.

Grimalsky, V. V., M. Hayakawa, V. N. Ivchenko, Y. G. Rapoport, and V. I. Zadorozhnii, Penetration of an electrostatic field from the lithosphere into ionosphere and its effect on the D-region before earthquakes, $J$. Atmos. Sol.-Terr. Phys., 65, 391-407, 2003.

Hayakawa, M., VLF/LF radio sounding of ionospheric perturbations associated with earthquakes, Sensors, 7, 1141-1158, 2007.

Hayakawa, M. and Y. Fujinawa, Electromagnetic Phenomena Related to Earthquake Prediction, Terra Sci. Publ. Co., Tokyo, 1994.

Hayakawa, M. and O. Molchanov, Seismo Electromagnetics Lithosphere-
Atomosphere-Ionosphere Coupling, Terra Sci. Publ. Co., Tokyo, 2002.

Hines, C. O., Internal atmospheric gravity waves at ionospheric heights, Can. J. Phys., 38, 1441-1481, 1960.

Karpov, I. V. and F. S. Bessarab, Model studying the effect of the solar terminator on the thermospheric parameters, Geomagn. Aeron., 48, 209219, 2008.

Klimenko, M. V. and V. V. Klimenko, Numerical simulation of the earthquake precursors in the ionosphere which are caused by electric fields, in Abstracts of 19th IAGA WG 1.2 Workshop on Electromagnetic Induction in the Earth, pp. 900-905, 23-29 October 2008, Beijing, China, 2008.

Klimenko, M. V., V. V. Klimenko, and V. V. Bryukhanov, Numerical simulation of the electric field and zonal current in the Earth's ionosphere: The dynamo field and equatorial electrojet, Geomagn. Aeron., 46, 457466, 2006.

Klimenko, M. V., V. V. Klimenko, and V. V. Bryukhanov, Numerical modeling of the equatorial electrojet UT-variation on the basis of the model GSM TIP, Adv. Radio Sci., 5, 385-392, 2007.

Klimenko, M. V., V. V. Klimenko, I. E. Zakharenkova, S. A. Pulinets, B. Zhao, and M. N. Tsidilina, Formation mechanism of great positive TEC disturbances prior to Wenchuan earthquake on May 12, 2008, Adv. Space Res., 48, 488-499, 2011.

Leonard, R. S. and R. A. Barnes, Observation of ionospheric disturbances following the Alaskan earthquake, J. Geophys. Res., 70, 1250-1253, 1965.

Liperovsky, V. A., O. A. Pokhotelov, and S. L. Shalimov, Ionospheric Precursors of Earthquakes, Moscow, Nauka, 1992 (in Russian).

Liperovsky, V. A., O. A. Pokhotelov, C.-V. Meister, and E. V. Liperovskaya, Physical models of coupling in the lithosphere-atmosphereionosphere system before earthquakes, Geomagn. Aeron., 48, 795-806, 2008.

Liu, J. Y., Y. I. Chen, Y. J. Chuo, and H. F. Tsai, Variations of ionospheric total electron content during the Chi-Chi earthquake, Geophys. Res. Lett., 28, 1383-1386, 2001.

Liu, J. Y., Y. J. Chuo, S. J. Shan, Y. B. Tsai, S. A. Pulinets, and S. B. Yu, Pre-earthquake ionospheric anomalies monitored by GPS TEC, Ann. Geophys., 22, 1585-1593, 2004.

Mareev, E. A., D. I. Iudin, and O. A. Molchanov, Mosaic source of internal gravity waves associated with seismic activity, in Seismo-Electromagnetics (Lithosphere-Atmosphere-Ionosphere Coupling), edited by Hayakawa, M. and O. Molchanov, pp. 335-342, Terra Sci. Publ. Co., Tokyo, 2002.

Miyaki, K., M. Hayakawa, and O. A. Molchanov, The role of gravity waves in the lithosphere-ionosphere coupling, as revealed from the subionospheric LF propagation data, in Seismo-Electromagnetics (LithosphereAtmosphere-Ionosphere Coupling), edited by Hayakawa, M. and O. Molchanov, pp. 229-232, Terra Sci. Publ. Co., Tokyo, 2002.

Molchanov, O. A., M. Hayakawa, and V. A. Rafalsky, Penetration characteristics of electromagnetic emissions from an underground seismic source into the atmosphere, ionosphere and magnetosphere, J. Geophys. Res., 100, 1691-1712, 1995.

Molchanov, O. A., On the origin of low- and middle-latitude ionospheric turbulence, Phys. Chem. Earth, 29, 559-567, 2004.

Namgaladze, A. A., Yu. N. Korenkov, V. V. Klimenko, I. V. Karpov, F. S. Bessarab, V. A. Surotkin, T. A. Glushchenko, and N. M. Naumova, Global model of the thermosphere-ionosphere-protonosphere system, Pure Appl. Geophys., 127, 219-254, 1988.

Namgaladze, A. A., Yu. N. Korenkov, V. V. Klimenko, I. V. Karpov, V. A. Surotkin, and N. M. Naumova, Numerical modelling of the thermosphere-ionosphere-protonosphere system, J. Atmos. Terr. Phys., 53, 1113-1124, 1991.

Namgaladze, A. A., M. V. Klimenko, V. V. Klimenko, and I. E. Zakharenkova, Formation of ionospheric earthquake precursors by electric field, in Collection of the Reports of IV International Conference "Solar-terrestrial Relations and Precursors of Earthquakes", pp. 424430, 14-17 August 2007, v. Paratunka, Kamchatka region, Russia, Petropavlovsk-Kamchatsky, IKIR, 2007 (in Russian).

Namgaladze, A. A., M. V. Klimenko, V. V. Klimenko, and I. E. Zakharenkova, Physical mechanism and mathematical simulation of ionosphere earthquake precursors observed in total electron content, Geomagn. Aeron., 49, 252-262, 2009.

Occhipinti, G., P. Lognonné, E. Alam Kherani, and H. Hébert, Three-dimensional waveform modeling of ionospheric signature induced by the 2004 Sumatra tsunami, Geophys. Res. Lett., 33, doi:10.1029/2006GL026865, 2006.

Occhipinti, G., E. Alam Kherani, and P. Lognonné, Geomagnetic depen- 
dence of ionospheric disturbances induced by tsunamigenic internal gravity waves, Geophys. J. Int., 173, 753-768, 2008.

Oraevsky, V. N., Yu. Ya. Ruzhin, and I. I. Shagimuratov, Anomalies of ionospheric TEC above Turkey before two strong earthquakes at 1999 , in Proceedings of 15th Wroclaw EMC Symposium, pp. 508-512, 27-30 June 2000, Wroclaw, Poland, 2000.

Oyama, K.-I., Y. Kakinami, J.-Y. Liu, M. Kamogawa, and T. Kodama, Reduction of electron temperature in low-latitude ionosphere at 600 $\mathrm{km}$ before and after large earthquakes, J. Geophys. Res., 113, A11317, doi:10.1029/2008JA013367, 2008.

Pertsev, N. N. and S. L. Shalimov, Generation of atmospheric gravitational waves in a seismo-active region and its influence on the ionosphere, Geomagn. Aeron., 36, 111-118, 1996.

Plotkin, V. V., GPS detection of ionospheric perturbation before the 13 February 2001, El Salvador earthquake, Nat. Haz. Earth Syst. Sci., 3, 249-253, 2003.

Pulinets, S. A., Lithosphere-Atmosphere-Ionosphere Coupling (LAIC) model, in Electromagnetic Phenomena Associated with Earthquakes, edited by M. Hayakawa, 235-254, 2009a.

Pulinets, S. A., Physical mechanism of the vertical electric field generation over active tectonic faults, Adv. Space Res., 44, 767-773, 2009b.

Pulinets, S. A. and K. Boyarchuk, Ionospheric Precursors of Earthquakes, Springer, Berlin, Germany, 2004

Pulinets, S. A., A. D. Legen'ka, T. V. Gaivoronskaya, and V. Kh. Depuev, Main phenomenological features of ionospheric precursors of strong earthquakes, J. Atmos. Sol.-Terr. Phys., 65, 1337-1347, 2003.

Rozhnoi, A., M. Solovieva, O. Molchanov, P.-F. Biagi, and M. Hayakawa, Observation evidences of atmospheric Gravity Waves induced by seismic activity from analysis of subionospheric LF signal spectra, Nat. Haz. Earth Syst. Sci., 7, 625-628, 2007.

Rulenko, O. P., Operative precursors of earthquakes in the near-ground atmosphere electricity, J. Volcanol. Seismol., 4, 57-68, 2000.

Sorokin, V. M. and V. M. Chmyrev, Modification of the ionosphere by seismic related electric field, in Atmospheric and Ionospheric Electromagnetic Phenomena Associated with Earthquakes, edited by Hayakawa, M., pp. 805-818, Terra Sci. Publ. Co., Tokyo, 1999.

Tzanis, A. and F. Vallianatos, A critical review of electric earthquake precursors, Ann. Geophys., 44, 429-460, 2001.

Yokoyama, T., T. Horinouchi, M. Yamamoto, and S. Fukao, Modulation of the midlatitude ionospheric $E$ region by atmospheric gravity waves through polarization electric field, J. Geophys. Res., 109, doi:10.1029/2004JA010508, 2004.

Zakharenkova, I. E., A. Krankowski, and I. I. Shagimuratov, Modification of the low-latitude ionosphere before December 26, 2004 Indonesian earthquake, Nat. Haz. Earth Syst. Sci., 6, 817-823, 2006.

Zakharenkova, I. E., I. I. Shagimuratov, and A. Krankowski, Features of the ionosphere behavior before Kythira 2006 earthquake, Acta Geophysica, 55(4), 524-534, 2007.

Zakharenkova, I. E., I. I. Shagimuratov, N. Yu. Tepenitzina, and A. Krankowski, Anomalous modification of the ionospheric total electron content prior to the 26 September 2005 Peru earthquake, J. Atmos. Sol. Terr. Phys., 70, 1919-1928, 2008.

Zolotov, O. V., A. A. Namgaladze, I. E. Zakharenkova, I. I. Shagimuratov, and O. V. Martynenko, Simulations of the equatorial ionosphere response to the seismic electric fields sources, in Proceedings of the 7th International Conference "Problems of Geocosmos", pp. 492-496, 2630 May 2008, St. Petersburg, Russia, St. Petersburg, St. Petersburg State University, 2008.

M. V. Klimenko (e-mail: maksim.klimenko@mail.ru), V. V. Klimenko, I. E. Zakharenkova, and I. V. Karpov 